\title{
Definition of Restenosis after Percutaneous Transluminal Coronary Angioplasty: A Quickly Evolving Concept
}

\author{
PATRICK W. SERRUYS, M.D., Ph.D., BENNO J. RENSING, M.D., \\ WALTER R.M. HERMANS, M.D., and KEVIN J, BEATT, M.D.
}

\section{Introduction}

Symptoms, Function, or Anatomy as Criteria of Restenosis? The incidence of late restenosis has remained much the same over the 13 years that angioplasty has been part of clinical practice and there is still no proven intervention that modifies the restenosis process. ${ }^{1}$

Primary success and restenosis after percutaneous transluminal coronary angioplasty (PTCA) may be defined by symptomatic criteria such as frequency and severity of anginal episodes, by functional criteria such as pressure flow characteristic of the dilated vessel, coronary flow reserve and various noninvasive diagnostic tests, or may be defined by anatomic criteria using postmortem histology, angiography, or intervascular ultrasound. These three criteria may be considered separately or may be interrelated so that the definition of restenosis becomes a complex issue. About the symptomatic criteria, it is fair to emphasize the four following points. (1) Although the subjective improvement of symptoms after PTCA is probably the most desirable endpoint, it is also the least objective evaluation. ${ }^{2}$ (2) The frequency of symptomatic improvement appears to be lower than that of angiographic success:

Address for reprints: Patrick W. Serruys, M.D., Ph.D., Catheterization Laboratory, Thoraxcenter, Erasmus University, P.O. Box 1738, 3000 DR Rotterdam, The Netherlands. Fax: 31-10-4365192.

Submitted for publication September 10, 1991; accepted September 23, 1991 only $80 \%-85 \%$ of the patients with a satisfactory angiography result immediately post-PTCA exhibit such an improvement. ${ }^{3}$ (3) The reappearance of angina as a sole criterion of restenosis underestimates the angiographic rate of restenosis. The reported incidence of silent restenosis may be as high as $33 \%$. (4) However, the elapsed time from PTCA to recurrence of symptoms has shown to be clinically useful in identifying the most probable cause of recurrent angina:

-Within 1 month: incomplete revascularization from additional coronary artery disease and/or incomplete dilatation should be suspected.

-Within 1-6 months: restenosis, that is to say, lesion recurrence is most likely.

-After 6 months: new significant atherosclerosis disease should be considered. ${ }^{4}$

The value of recurrent anginal symptoms as a marker of restenosis is difficult to assess in many studies because the timing and completeness of angiographic follow-up often have been determined by symptomatic status. In studies with a high rate of angiographic follow-up, the probability that patients with symptoms had restenosis (i.e., the positive predictive value of symptoms) ranged from $48 \%-92 \%$, whereas the probability that patients without symptoms were free of restenosis (i.e., the negative predictive value of symptoms) range from $70 \%-98 \%$ (Table 1 ). ${ }^{5}$

The low positive predictive value found in many of these studies may be explained by the presence of other mechanism for angina, such as incomplete revascularization or progress of disease in other vessels. 
Table 1. Detection of Restenosis by Symptoms

\begin{tabular}{lccccc}
\hline & & & & \multicolumn{3}{c}{ Symptoms } \\
\cline { 5 - 6 } Author & Year & $\begin{array}{c}\text { Anglographic } \\
\%\end{array}$ & $\begin{array}{c}\text { Restenosis } \\
\%\end{array}$ & $\begin{array}{c}\text { PPV } \\
\%\end{array}$ & $\begin{array}{c}\text { NPV } \\
\%\end{array}$ \\
\hline Simonton & $' 88$ & 90 & 35 & 48 & 75 \\
Califf & $' 90$ & 100 & 38 & 60 & 85 \\
Zaidi & $' 85$ & 100 & 49 & 66 & 70 \\
Mabin & $' 85$ & 55 & 32 & 71 & 86 \\
Levine & $' 85$ & 92 & 40 & 76 & 96 \\
Jutzy & $' 82$ & 88 & 47 & 92 & 83 \\
Gruentzig & $' 87$ & 93 & 31 & 92 & 98 \\
\hline
\end{tabular}

Modified from Califf et al. ${ }^{5} \mathrm{PPV}=$ positive predictive value; $\mathrm{NPV}=$ negative predictive value

Several studies have examined the ability of the exercise treadmill test to detect restenosis after PTCA. These studies have generally found that the presence of exercise-induced angina or ST segment depression or both is not highly predictive of restenosis whether the test is performed early or late after angioplasty. The positive predictive values of early treadmill testing range from $29 \%-60 \%$, whereas the corresponding values for late treadmill testing are ranging from $39 \%-64 \%$ (Table 2). The low positive predictive value is most likely a consequence of incomplete revascularization: that is either a totally occluded vessel, or a significant stenosis at a site other than dilated by angioplasty. It is also possible that the noninvasive test is accurately demonstrating a functionally inadequate dilatation, despite the appearance of success on angiography.
Table 3 shows the accuracy of thallium scintigraphy for detection of restenosis in series, which have a reasonable angiographic follow-up ranging from $55 \%-100 \%$. Since cardiac catherization remains "the gold standard" for detection of restenosis, the reported value of a noninvasive tests is determined not only by the actual accuracy of the test but also by the completeness of angiographic follow-up. In these studies with a high rate of angiographic follow-up the positive predictive value of thallium scintigraphy is ranging between $56 \%$ and $89 \%$.

Recently, Lefkowitz et al. ${ }^{6}$ have shown that the positive and negative predictive values for tomographic imaging in detection of restenosis were superior to the predictive values observed with planar imaging. In addition, the specific vascular territory was correctly localized to the PTCA territory in $77 \%$ of the tomographic studies.

Coronary Angiography Still "the Gold Standard". In view of the above, coronary angiography still is the most reliable method of judging the late results. Unfortunately, there are many studies on coronary restenosis reported that are distinguished by their lack of consistency in their methodological approach and their definitions of restenosis. Figure 1 illustrates this point. On the vertical axis of this "nonscientific" figure, we have the names of the investigators, who have studied the restenosis problem, on the horizontal axis we have the restenosis rate observed in their studies. A restenosis rate ranging between $25 \%$ and $35 \%$ seems to emerge. However, we have to

Table 2. Detection of Restenosis by Exercise Treadmill Testing

\begin{tabular}{lccccc}
\hline Author & $\begin{array}{c}\text { Angiographic } \\
\text { Follow-Up } \\
\%\end{array}$ & $\begin{array}{c}\text { Restenosis } \\
\%\end{array}$ & $\begin{array}{c}\text { PPV } \\
\%\end{array}$ & $\begin{array}{c}\text { NPV } \\
\%\end{array}$ & Timing of Test \\
\hline O'Keefe & 100 & 13 & 29 & 73 & $<1$ month \\
Scholl & 83 & 12 & 40 & 27 & 1 month \\
Wijns* & 74 & 35 & 50 & 65 & 3-7 weeks \\
Wijns* & 89 & 40 & 60 & 52 & $3-8$ weeks \\
Bengston & 96 & 51 & 39 & 84 & 6 months \\
Rosing & 100 & 34 & 47 & 76 & 8 months \\
Ernst & 100 & 4 & 50 & 95 & 4-8 months \\
Honan & 88 & 58 & 57 & 64 & 6 months \\
Scholl & 83 & 12 & 64 & 50 & 6 months \\
\hline
\end{tabular}

Modified from Califf et al. ${ }^{5} \mathrm{PPV}=$ positive predictive value; $\mathrm{NPV}=$ negative predictive value .

* Thoraxcenter. 


\section{DEFINITION OF RESTENOSIS AFTER PTCA}

Table 3. Detection of Restenosis by Thallium 201 Scintigraphy

\begin{tabular}{lccccc}
\hline Author & $\begin{array}{c}\text { Angiographic } \\
\text { Follow-Up } \\
\%\end{array}$ & $\begin{array}{c}\text { Restenosis } \\
\%\end{array}$ & $\begin{array}{c}\text { PPV } \\
\%\end{array}$ & $\begin{array}{c}\text { NPV } \\
\%\end{array}$ & Timing of Test \\
\hline Jain & 55 & 14 & 79 & 88 & $0-6$ days \\
Miller & 76 & 39 & 76 & 94 & 2 weeks \\
Lam & 100 & 9 & 89 & 96 & 2 weeks \\
Wijns* & 74 & 35 & 74 & 83 & $3-7$ weeks \\
Wijns* & 89 & 40 & 82 & 72 & $3-8$ weeks \\
Scholl & 83 & 12 & 56 & 42 & 1 months \\
& & & 100 & 75 & 6 months \\
Ernst & 100 & 4 & 50 & 100 & $4-8$ months \\
Rosing & 100 & 21 & 37 & 83 & 8 months \\
Lefkowitz & planar thallium & & 62 & 80 & 6 months \\
& tomographic thallium & & 80 & 93 & \\
\hline
\end{tabular}

Modified from Califf et al..$^{5}$ PPV $=$ positive predictive value; NPV $=$ negative predictive value.

* Thoraxcenter

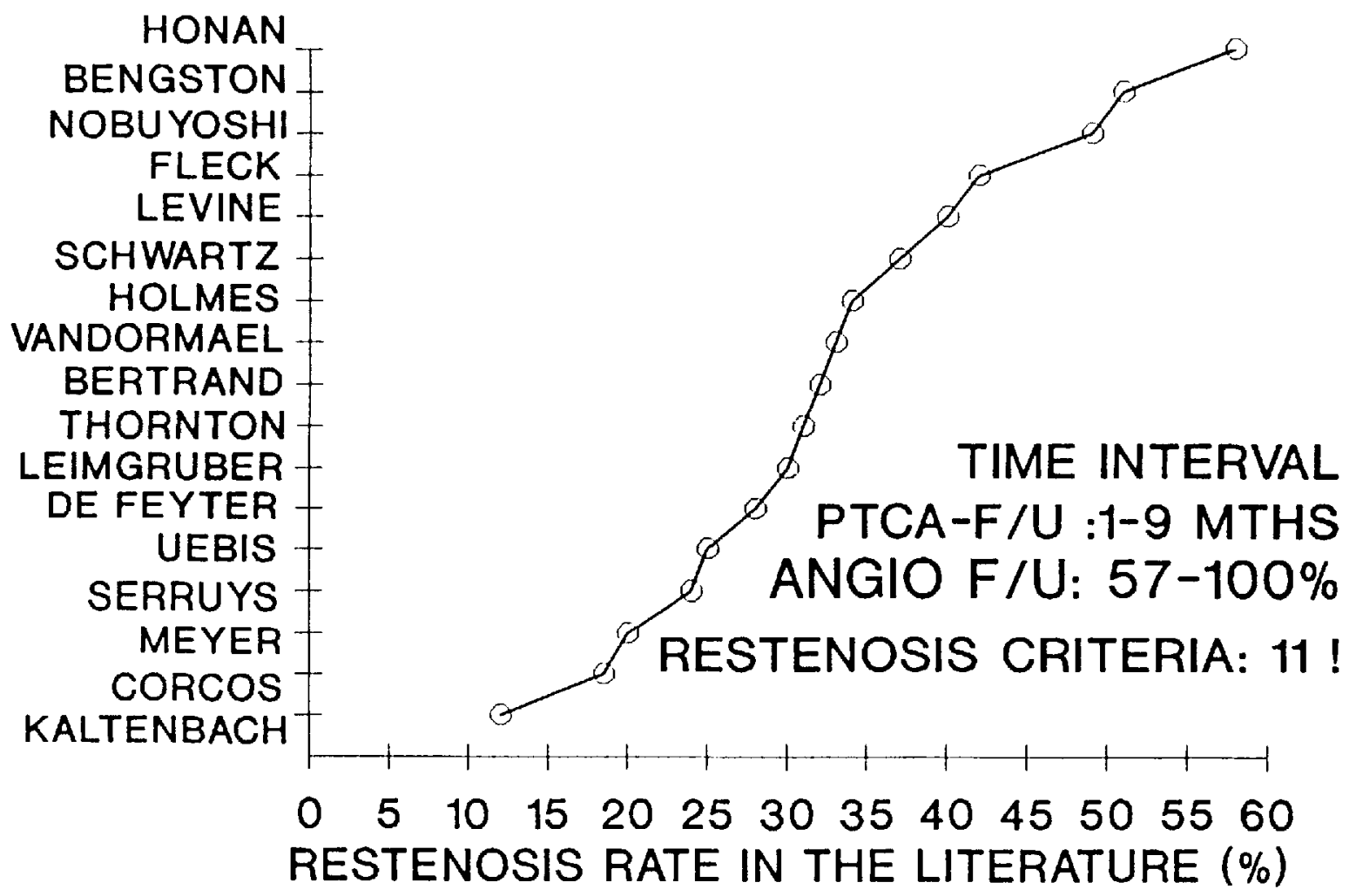

Figure 1. Restenosis rates found by different authors, applying 11 different restenosis criteria, different angiographic followup (F/C) times (1-9 months), and different analysis techniques (visual or quantitative). 


\section{SERRUYS, ET AL.}

Table 4. Criteria of Restenosis in Current Use

1. Loss of at least $50 \%$ of the initial gain (NHLBI 4)

2. A return to within $10 \%$ of the pre-PTCA diameter stenosis (NHLBI 3)

3. An immediate post-PTCA $<50 \%$ diameter stenosis that increases to $\geq 50 \%$ at follow-up

4. As for 3 , but for a diameter stenosis $\geq 70 \%$ at follow-up (NHLBI 2)

5. Reduction $\geq 20 \%$ in diameter stenosis

6. Reduction $\geq 30 \%$ in diameter stenosis (NHLBI 1 )

7. A diameter stenosis $\geq 50 \%$ at follow-up

8. A diameter stenosis $\geq 70 \%$ at follow-up

9. Area stenosis $\geq 85 \% 14$

10. Loss $\geq 1 \mathrm{~mm}^{2}$ in stenosis area ${ }^{15}$

11. Deterioration of $\geq 0.72 \mathrm{~mm}$ in minimal luminal diameter from post-PTCA to follow-up ${ }^{13}$

12. Deterioration of $\geq 0.5 \mathrm{~mm}$ in minimal luminal diameter from post-PTCA to follow-up ${ }^{12}$

NHLBI $=$ National Heart Lung Blood Institute; PTCA = percutaneous transluminal coronary angioplasty

emphasize the following facts: the angiographic follow-up in these patients range between $57 \%$ and $100 \%$, the time to follow-up range between 1 and 9 months, and 11 different criteria of restenosis have been applied by these investigators who in large majority used visual assessment of the coronary angiogram.

The variety of criteria in current use is tabulated in Table 4. Most are entirely arbitrary, some are based on doubtful logic and some, although of some relevance for visual estimation of percent diameter stenosis, are unrealistic when applied to the most accurate values obtained from quantitative angiography. Thus, most of the discrepancies between these studies can be attributed to three factors: (1) the selection of patients, (2) the method of analysis, and (3) the definition of restenosis used. In order to improve the situation these three factors need to be addressed.?

1. The study population. This means a high angiographic follow-up rate $(>80 \%)$ with a predetermined time for restudy, this will avoid a selection bias of symptomatic patient. Sample size of observational or randomized clinical trial should be adequately controlled to avoid a type II error commonly referred to as the power of the test.

2. A well validated system of analysis with known accuracy and variability should be used. The use of a visual percent diameter stenosis measurement with its inherent variability precludes meaningful results and edge tracing by hand or other techniques that can produce values not physiologically possible are also unacceptable. ${ }^{8}$ Video densitometry may eventually provide the best measurement because the technique estimates the volume of the lumen independently of geometric assumption, but for technical reason this theoretical method of choice has not (yet) proven practical. ${ }^{9}$

3. The measured variables must be chosen so as to reflect the restenosis proliferative process and distinguish between the results of angioplasty (optimal or suboptimal) and this proliferative restenosis process. We believe that the conventional assessment of percent diameter stenosis is not sufficiently discriminating in doing this and that definitions based on percent diameter stenosis measurement fail to identify lesions undergoing significant deterioration. ${ }^{7,10}$ Percent diameter stenosis criteria are chosen to reflect the change in minimal luminal diameter in relation to the so-called normal diameter of the vessel in the immediate vicinity of the obstruction. In the first place it is assumed that there is a normal diameter; diffuse intimal or subintimal thickening are not detectable on a coronary "shadowgram." Second, the choice of a so-called normal diameter, proximal or distal to the obstruction is arbitrary and will have major impact on the calculation of the percentage diameter stenosis. This is illustrated in Figure 2; in this particular example the percentage diameter stenosis is ranging from $75 \%-60 \%$ according to the site of reference arbitrarily chosen. Third, it is assumed that this normal diameter does not change as a result of angioplasty or during the immediate follow-up period when restenosis of the dilated lesion is a well recognized phenomenon. The so-called normal diameter might be diffusely affected by the barotrauma of the balloon that can induce a reactive hyperplasia in the area touched by the balloon. ${ }^{10}$ This seriously questions the use of percent diameter stenosis as the only index of restenosis (Fig. $3)$.

Restenosis Definition, Subject to Debate. The restenosis definition of choice has been the subject of much debate and there is currently no satisfactory definition that takes into account the func- 


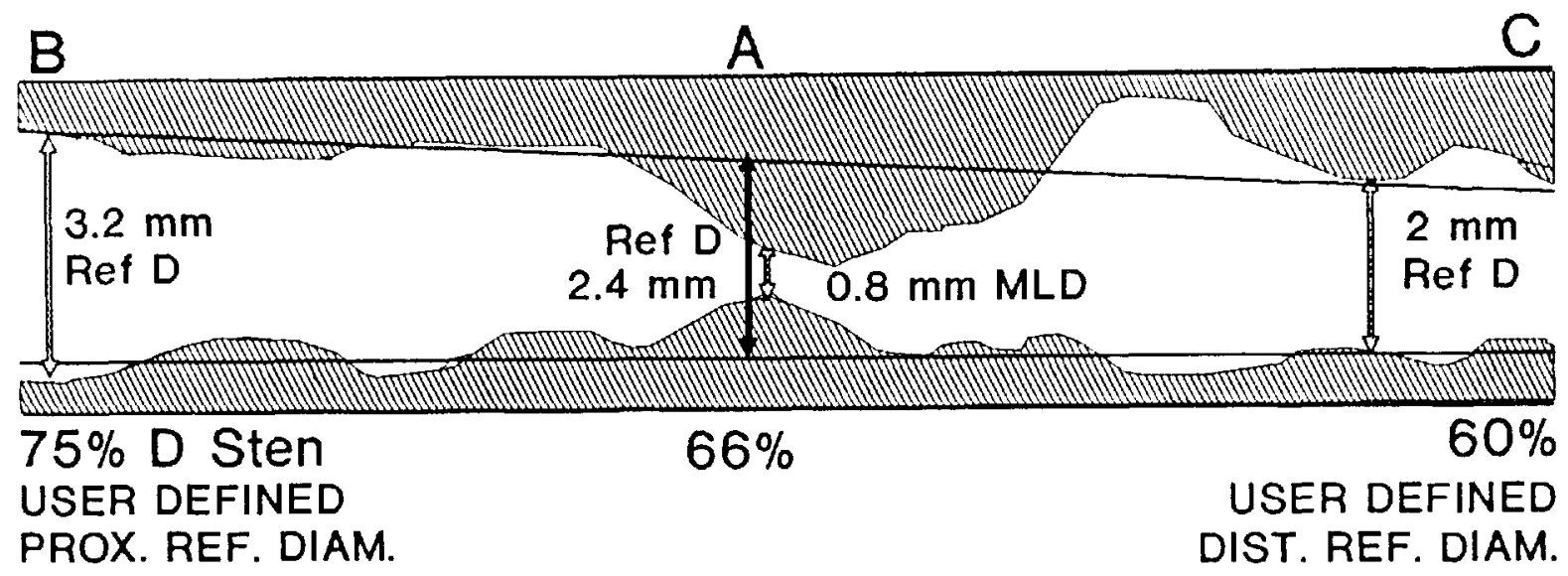

Figure 2. Graphical illustration of different percentage diameter stenosis values dependent on the arbitrary choice of the site of the reference diameter. With our quantitative analysis system it is possible to obtain an objective, independent value for the reference diameter and thus, for the percent diameter stenosis. This is called the "interpolated reference diameter." $A$ computer derived reconstruction of the original arterial dimension at the site of obstruction (assuming there is no disease present) is used to define the interpolated reference diameter (tapering lines in figure).

tional and angiographic outcome after PTCA. The known discrepancy between these two parameters means that this objective will not be realized. A single "stenosis" measurement should not be confused with a measurement of "restenosis," which should represent the change in stenosis severity. The commonly used definition of $50 \%$ diameter stenosis at follow-up is historically based on the physiological concept of coronary flow reserve introduced by Lance Gould " in 1974 and is taken because it represents the approximate value in animals with normal coronary arteries at which blunting of the hyperemic response occurs. Although this value may be of some relevance in determining a significant stenosis in human atherosclerotic vessels, it tells us nothing about the way the lesion has behaved since the angioplasty procedure.

In 1988, two different studies, performing follow-up angiography at different preselected follow-up intervals, gave remarkably similar results and showed more precisely how lesions behave after angioplasty. ${ }^{12,13}$ In a study carried out at the Thoraxcenter the minimal lumen diameter measured increased slightly from $2.06 \mathrm{~mm}$ to $2.11 \mathrm{~mm}$ at 30 days and then decreased steadily to 1.93 , 1.77 , and $1.69 \mathrm{~mm}$ at the subsequent follow-up times (2, 3, and 4 months). Nobuyoshi and colleagues restudied 229 patients at 24 hours, and 1 ,
3,6 , and 12 months. Their findings were very similar to ours (Fig. 4). In addition, it should be stressed that the individual changes in minimal luminal diameter of these lesions show that it is not just a limited number of lesions that "restenose," but rather almost all lesions deteriorate to some extent. This is a concept that is not well understood in the context to restenosis. A significant deterioration is also seen in the decreasing reference diameter that tends to minimize the change in the calculated percentage diameter stenosis. Furthermore, lesion progression after 6 months is unusual. ${ }^{12}$

While accurate (quantitative) assessment suggests that the trend to restenosis applies to most dilated lesions, deciding which of these lesions should be defined as restenosis is less clear. Indeed, the factor that most influences the restenosis rate is the definition of restenosis applied. Figure 5 shows the incidence of restenosis according to three criteria taken from a group of 490 lesions analyzed in the first 150 days after angioplasty $;^{13}$ the three criteria are the National Heart Lung Blood Institute criterion 4: a loss of greater than half the gain, a diameter $>50 \%$ at follow-up and a change $>0.72 \mathrm{~mm}$ from postangioplasty to follow-up. From this figure two conclusions can be drawn: first, there is a variation in the incidence of restenosis according to the criterion applied, 
USER DEFINED

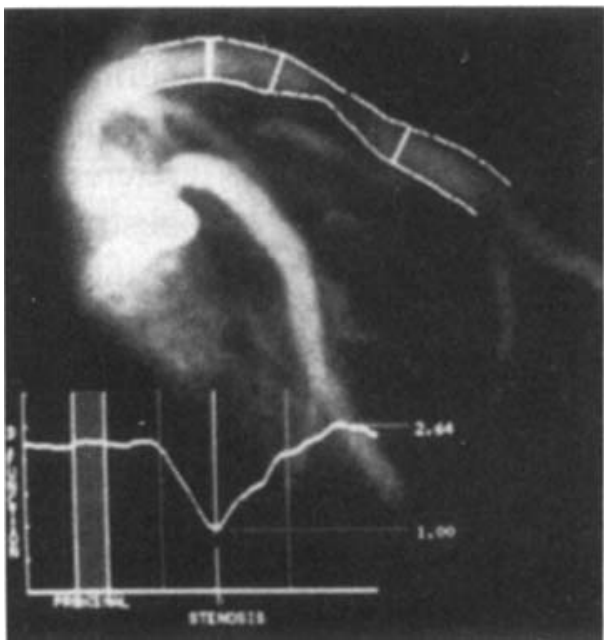

USER DEFINED

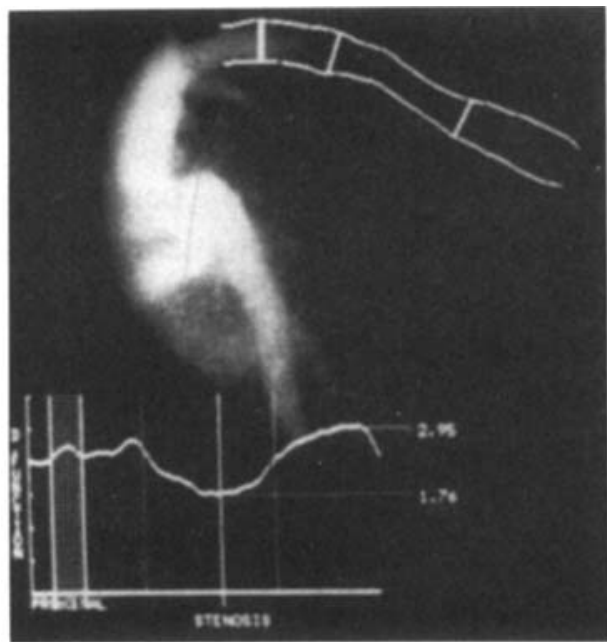

USER DEFINED PROXIMAL

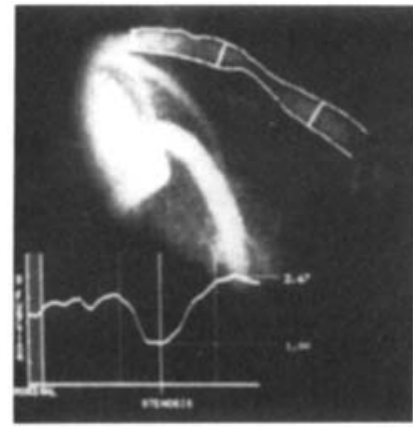

USER DEFIMED DISTAL
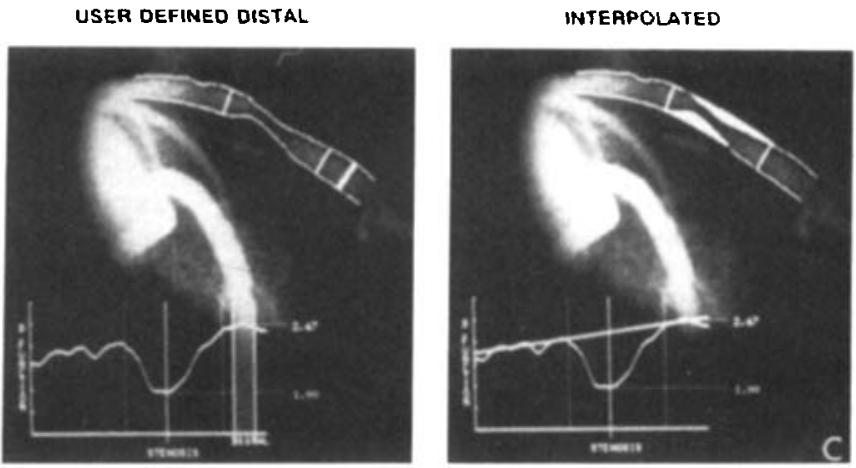

Figure 3. Single frame angiograms of a proximal left anterior descending artery stenosis. (A) Predilation (pre-PTCA), (B) postdilation (post-PTCA), and (C) at follow-up. Quantitative coronary analysis was performed using a coronary angiography analysis system. The arterial boundaries detected by the system are shown on the angiogram and below the diameter function curve derived from these contours. The example 


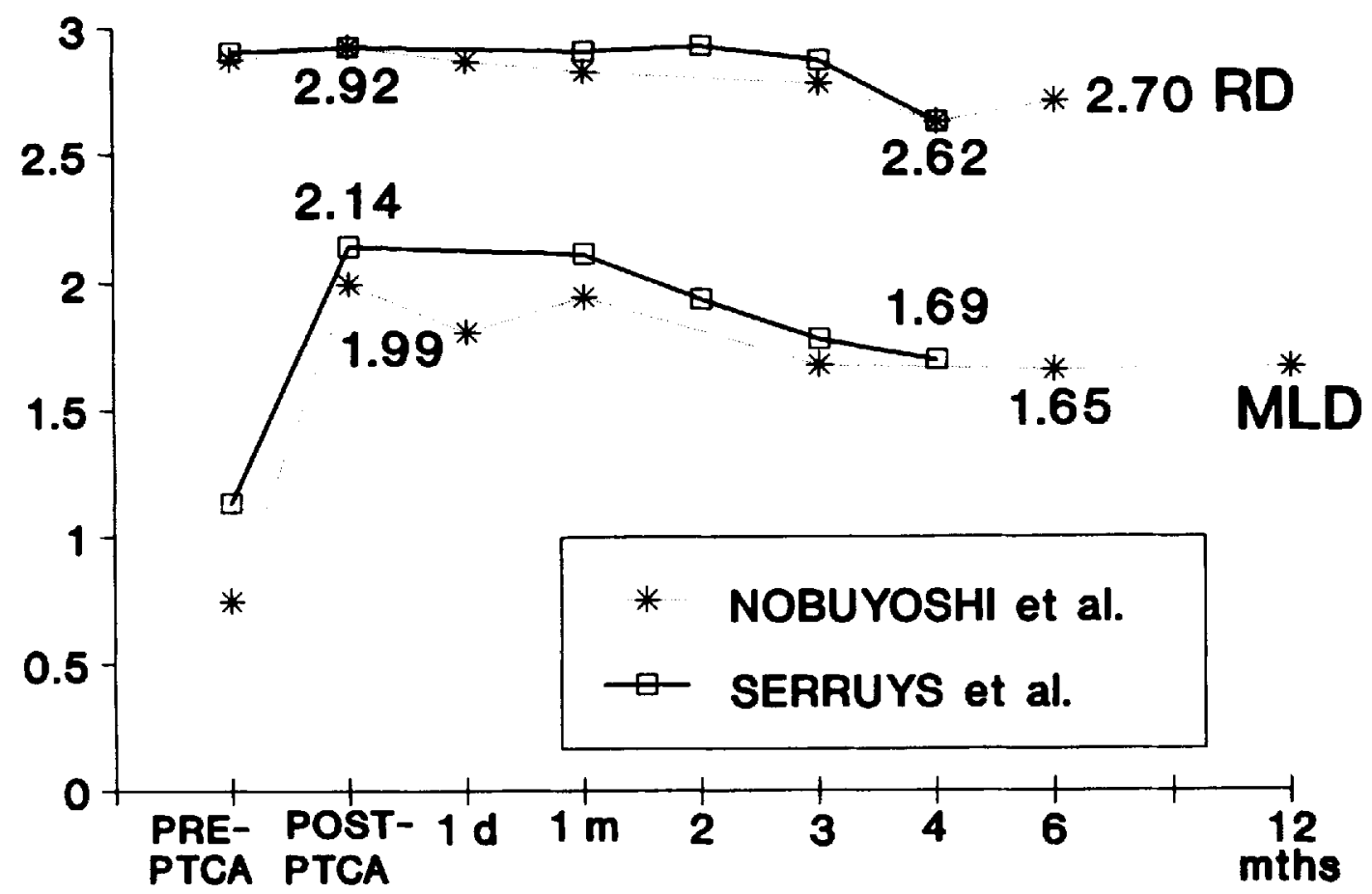

Figure 4. Minimal luminal diameter (MLD) and reference diameter (RD) values found in the studies by Nobuyoshi et al. ${ }^{12}$ and Serruys et al. ${ }^{13}$ Results are remarkably similar.

and second, the incidence of restenosis is progressive to at least the third month. At 5 months, the incidence of restenosis is not too dissimilar, ranging from $21 \%-34 \%$. However, it should be clear that even a similar incidence of restenosis using different criteria may be defining different patients. This is illustrated by Figure 6 , which shows a Venn diagram of the number of lesions fulfilling three different restenosis criteria, taken from the same group of 490 lesions. ${ }^{13}$ The 43 lesions that fulfill each criterions are enclosed by all three circles. Although the percentages of lesion, fulfilling the three criteria for restenosis were similar, it must be emphasized that each of the three criteria identified unique lesions that were not identified by the other two. This point has a par-

illustrates the importance of the choice of reference diameter, the fact that the dilated but nonstenotic coronary artery may be involved in the restenosis process, and the value of the interpolated reference diameter for calculating the appropriate diameter stenosis. (A) Before angioplasty, the lesion is relatively easy to analyze. The segments proximal and distal to the stenosis are of similar caliber and the lesion is relatively discrete, so that its length can easily be defined on the diameter function curve. (B) After angioplasty, there is a satisfactory result, the diameter stenosis decreasing from $59 \%$ to $36 \%$ (area stenosis from $83 \%$ to $59 \%$ ). (C) At follow-up, the result is dependent on the method of analysis. The artery proximal to the stenosis has already been involved in the restenosis process; if this is chosen as a reference diameter (left), a $42 \%$ diameter stenosis is obtained (no "restenosis"). The distal portion is of a larger caliber than the proximal portion; if it is chosen as a reference diameter (middle), the result is a $62 \%$ diameter stenosis ("restenosis"). If the interpolated technique is used (right), the reference diameter is similar to the postangioplasty value, and a $58 \%$ diameter stenosis is obtained that accurately reflects what is happening between the postangioplasty result and the follow-up. Even with this high quality angiogram of a well visualized segment with a discrete stenosis, there are problems in obtaining accurate and realistic results. 


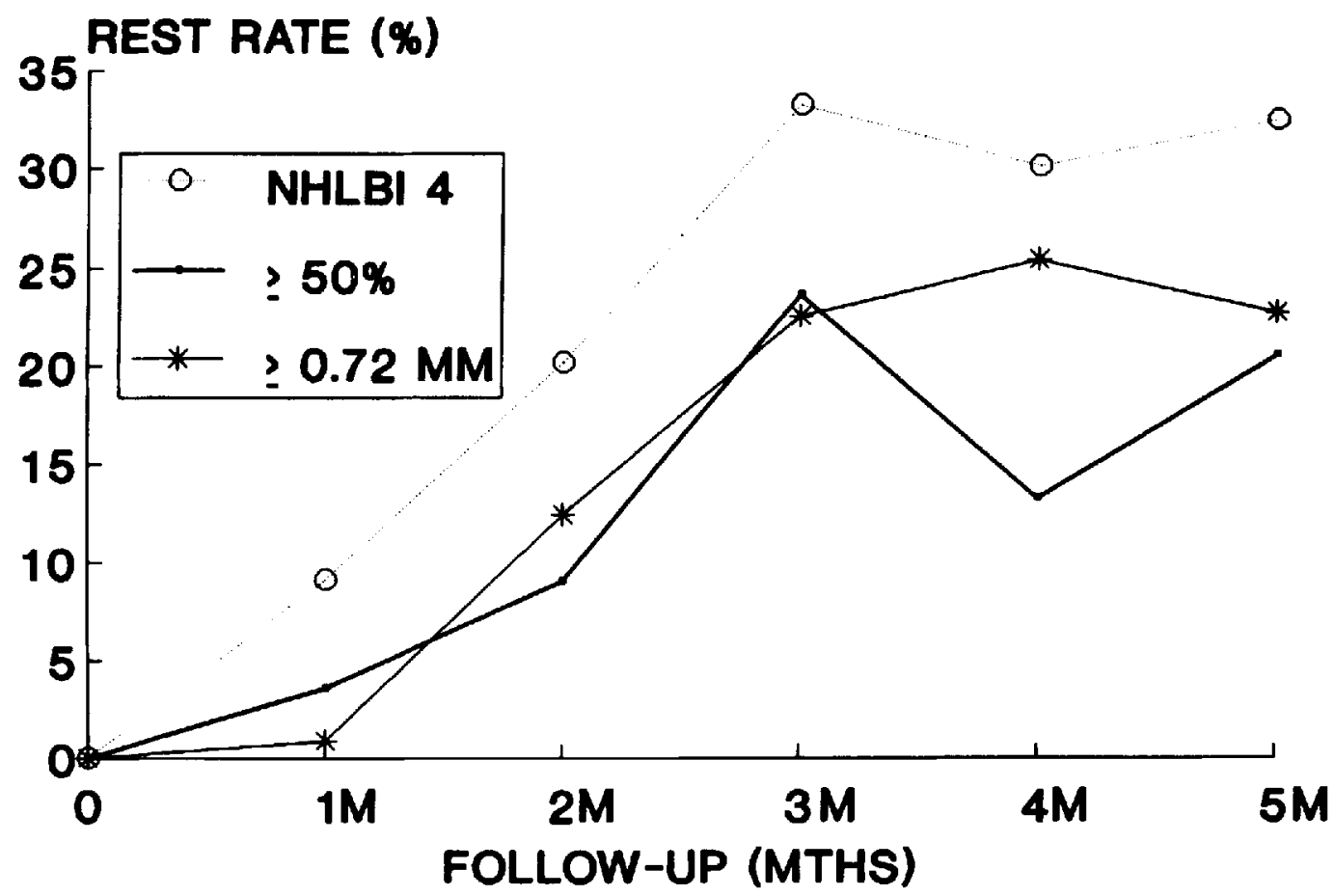

Figure 5, Restenosis rates at 1, 2, 3, 4, and 5 months (M) follow-up after PTCA, applying three different restenosis criteria. ${ }^{13}$ NHLBI 4 = National Heart Lung Blood Institute criterion 4, REST RATE $=$ restenosis rate.

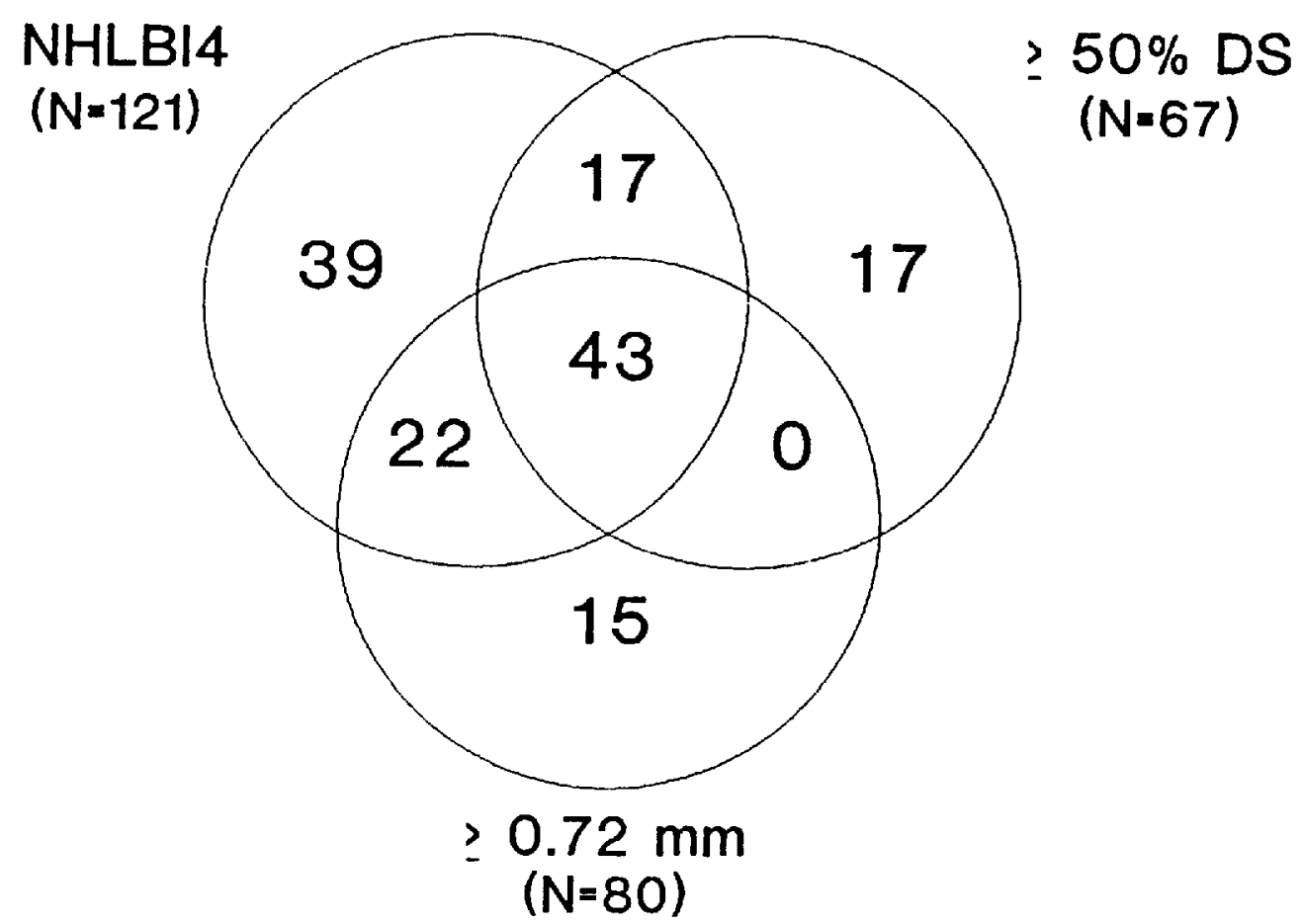

Figure 6. Venn diagram showing the number of lesions fulfilling three different restenosis criteria. ${ }^{12}$ 


\section{DEFINITION OF RESTENOSIS AFTER PTCA}

ticular relevance when determining the risk factors for restenosis: if restenosis cannot be reliably determined, then it is unlikely that the associated risk factors will be identified.

Restenosis Definition, New Concept. As a result of quantitative angiographic studies, a new concept for defining restenosis criteria based on the change in minimal lumen diameter has been introduced. The changes in this value from post-PTCA to follow-up can be expected to give a good quantitative measurement of the degree of restenosis. The restenosis criterion or the cutoff point dividing the restenosis patients from the nonrestenosis patient is then derived by determining the variability of measurements ( 1 standard deviation of the difference in means) of the same lesion from separate catheterization sessions. Twice the variability defines with reasonable certainty those lesions that have undergone significant deterioration from those that have not. We found this value to be $0.72 \mathrm{~mm}$ based on angiograms taken 90 days apart, whereas Nobuyoshi et al. ${ }^{12}$ using a different measurement system, found $0.5 \mathrm{~mm}$ based on angiograms taken 7-10 days apart. However, criteria based on the absolute change in minimal luminal diameter are nevertheless limited because they make no attempt to relate the extent of the restenosis process to the size of the vessel. Studies need to be undertaken to assess the var: ability of measurement on vessels with differeni diameters. "Sliding-scale" criteria should be created, which adjust for vessel size.

Restenosis Definition, a Categorical or Continuous Approach? In studies evaluating the biology of restenosis, a continuous measure of the degree of luminal obstruction is preferable since any progression of the stenosis reflects the process of interest whether or not an arbitrarily defined threshold of obstruction is reached. However, when the main concern is clinical decision mak-

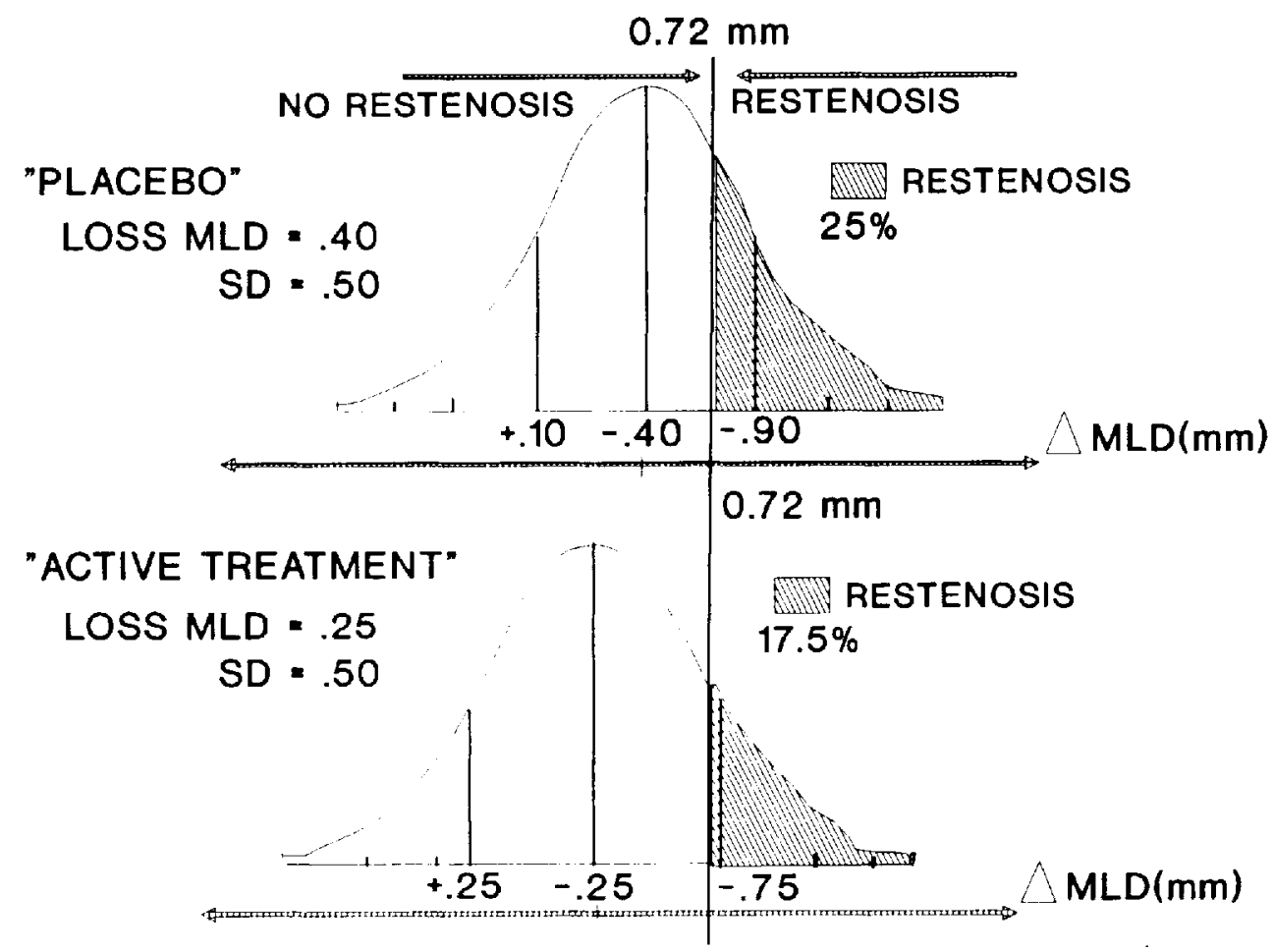

Figure 7. Gaussian model of restenosis rates in the reference group (upper panel) and in the treatment group (lower panel), considering a $30 \%$ reduction in minimal luminal diameter (MLD) change in the second group. The upper panel denotes the change in minimal lumen diameter found in a prospective study of our institutions. ${ }^{12}$ A change of $0.72 \mathrm{~mm}$ was taken as the cutoff point for restenosis. This categorical model would mean 620 patients per group in order to have a power of $90 \%$. 
ing, a binary or categorical measure of restenosis provides clinicians with more relevant information. Keeping in mind that an angiographic restenosis study assesses only the anatomical component of the restenosis problem, there is no threshold above which a loss of luminal diameter would have clinically significant functional or symptomatic consequences. Why then would one bother to try to define a threshold above which then would be "significant" quantitatively determined angiographic restenosis. To define the threshold on consideration of reproducibility of the measurement in individual patients is also questionable. The possible benefit of a treatment (pharmacological or interventional) can be measured with much greater precision by using the change in lumen diameter for the group. If treatment reduces the loss of luminal diameter from $0.4 \mathrm{~mm}$ under placebo ${ }^{13}$ to $0.25 \mathrm{~mm}$ under active medication, 233 patients per treatment group are required in order to detect a significant difference with a power of $90 \%$. The above reduction corre- sponds with restenosis rates (defined as a loss of minimal luminal diameter of $\geq 0.72 \mathrm{~mm}$ ) of $25 \%$ and $17.5 \%$, respectively (Fig. 7). This difference, however, can statistically be detected with a power of $90 \%$ with 620 patients per treatment group. Thus, statistically, the quantitative outcome determined from direct measurements of continuous variables can be evaluated with only one third of the number of patients required for the categorical outcome. This is indeed logical because the categorical end points do not take full advantage of the available information. Currently, the results of six randomized trials, involving 76 cities and more than 2,500 patients, are analyzed at the quantitative angiographic core laboratory in Rotterdam and at the data center in Geneva. In the future you might expect that the quantitative angiographic results will be presented according to a Gaussian model. Figure 8 shows the distribution of the change in minimal lumen diameter (MLD) from postangioplasty to 6 months follow-up angiography, excluding lesions

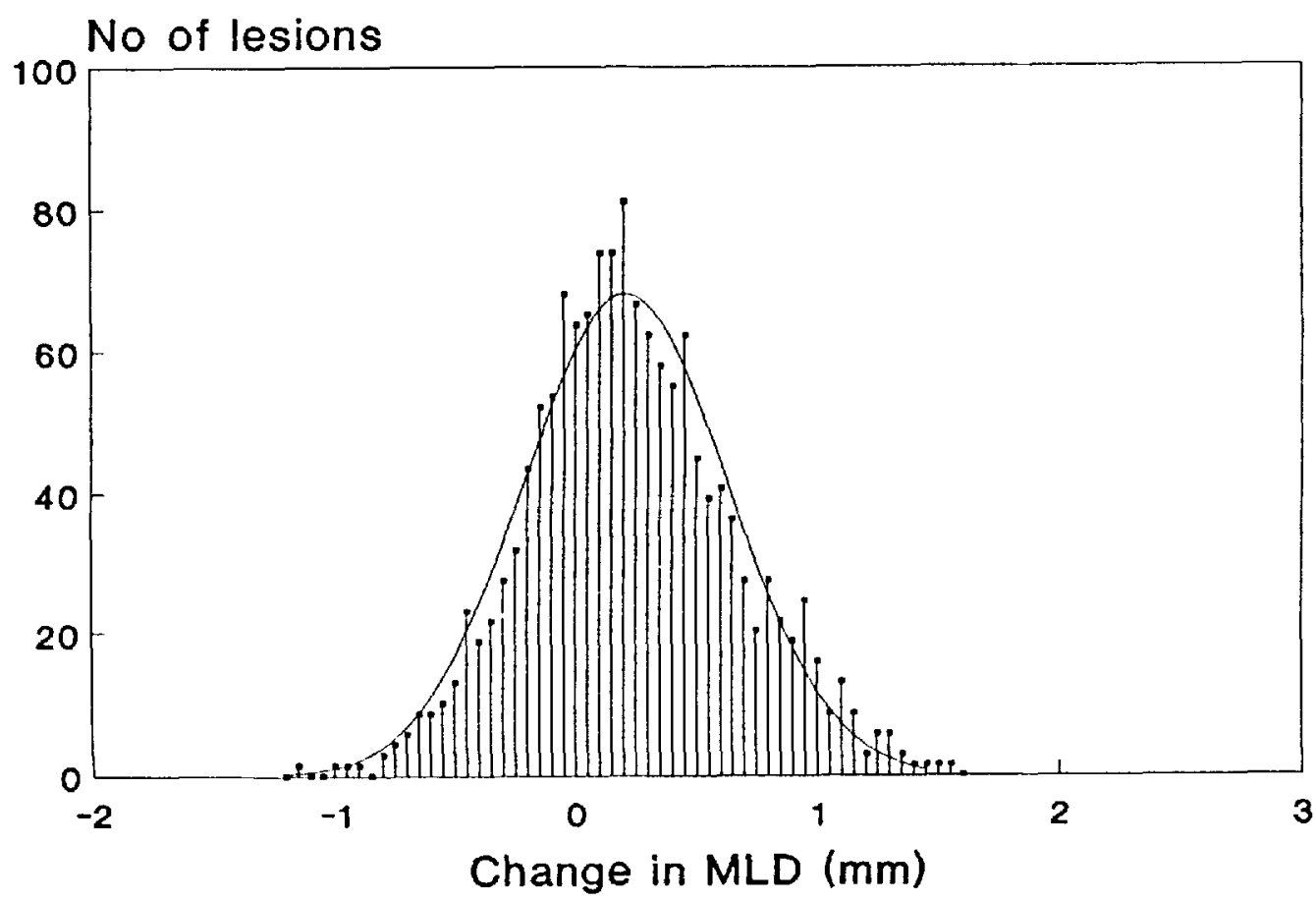

Figure 8. Distribution of the change in minimal lumen diameter (MLD) from postangioplasty angiogram to 6 months follow-up angiography in 1,375 lesions, excluding lesions that had progressed to total occlusion at follow-up. The curve superimposed on the distribution depicts the theoretical Gaussian distribution given the mean and standard deviation of the population. A change $>0$ corresponds with a decrease in MLD. 


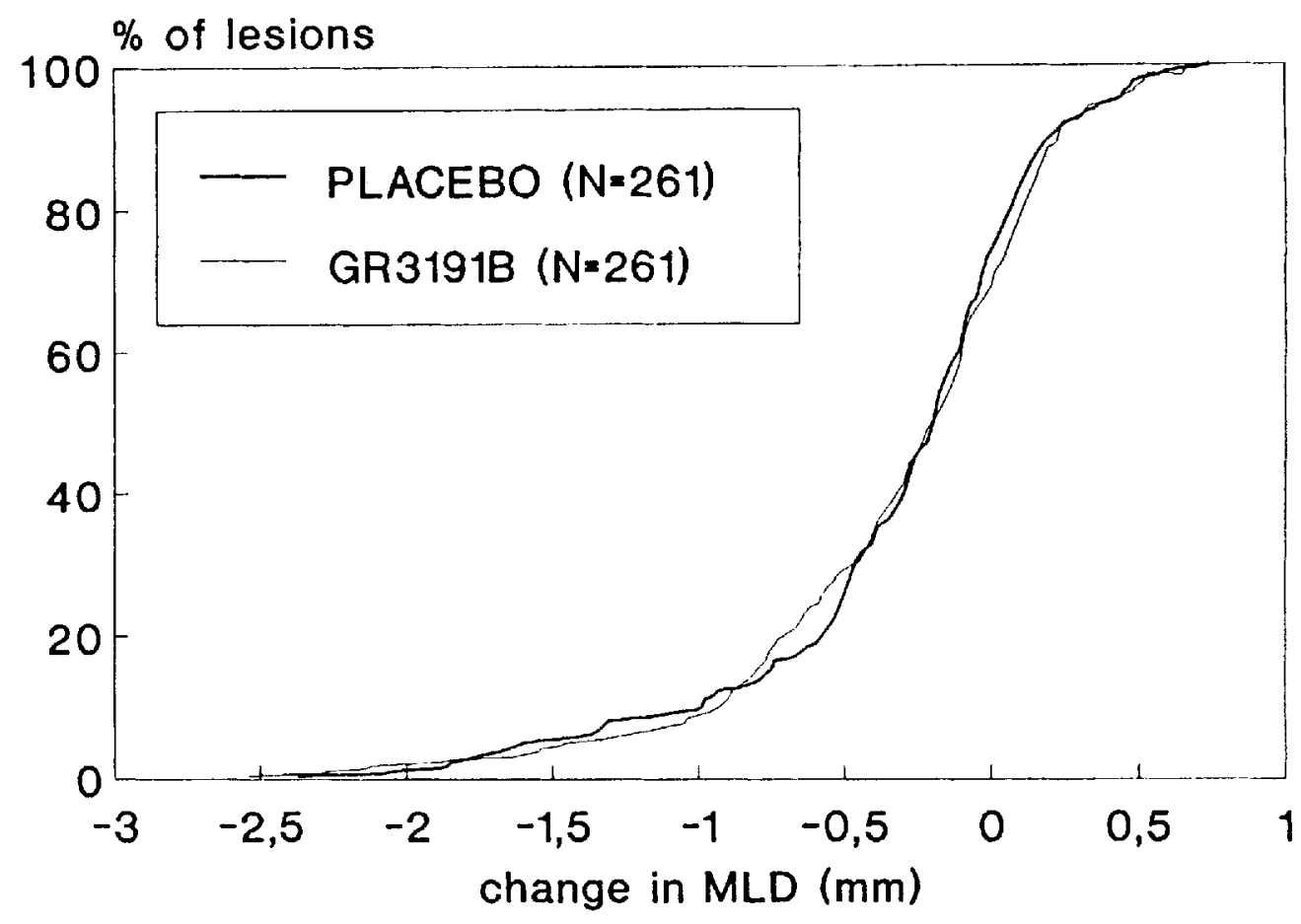

Figure 9. Cumulative distribution curve of the change in minimal lumen diameter (MLD) from postangioplasty angiogram to follow-up angiography for the placebo and the treatment group in a restenosis prevention trial.

that progressed to total occlusion. Superimposed is the theoretical Gaussian distribution curve, given the mean and standard deviation. It is clear that the change in MLD approximately follows this Gaussian distribution. Restenosis can thus be viewed as the tail end of an approximately Gaussian distributed phenomenon with some lesions crossing a more or less arbitrary cutoff point. A cumulative distribution curve of change in MLD is an clegant way of showing results of restenosis trials. Figure 9 shows this for a restenosis prevention trial with a Thromboxane $A_{2}$ receptor blocker.

In summary, with the advent of new technology of quantitative assessing coronary lesions, one should fully exploit the quantitative information (angiographic or ultrasonic) available (i.e., continuous approach.) This is particularly important in view of the fact that the quantitatively measured anatomical changes do not specifically relate to functional end points.
Acknowledgment: We gratefully acknowledge the skillful secretarial assistance of Hanneke Roerade and technical assistance of Marie-Angèle Morel in the preparation of the manuscript.

\section{References}

1. Serruys PW, Rensing BJ, Luijten HE, et al. Restenosis following coronary balloon angioplasty. In Meier B, ed. Interventional Cardiology. Toronto: Hogrefe \& Huber, 1990:79.

2. Block PC. Percutaneous transluminal coronary angioplasty: Role in the treatment of coronary artery disease. Circulation 1985; 72(suppl V): V-161-V-165.

3. Kent KM, Bonow RO, Rosing DR, et al. Improved myocardial function during exercise after successful percutaneous transluminal coronary angioplasty. N Engl J Med 1982; 306:441-446.

4. Joelson J, Most AS, Williams DO. Angiographic findings when chest pain recurs after successful percutaneous transluminal coronary angioplasty. Am J Cardiol 1987; $60: 792-795$. 


\section{SERRUYS, ET AL.}

5. Califf RM, Ohman EM, Frid DJ, et al. Restenosis: The clinical issues. In: Topol EJ, ed. Textbook of Interventional Cardiology. Philadelphia: W.B. Saunders, 1990:363-394.

6. Lefkowitz CA, Ross BL, Schwartz L, et al. Superiority of tomographic thallium imaging for the detection of restenosis after percutaneous transluminal coronary angioplasty (abstract). J Am Coll Cardiol 1988; 13:161 A.

7. Beatt KJ, Serruys PW, Hugenholtz PG. Restenosis after coronary angioplasty: New standards for clinical studies. J Am Coll Cardiol 1990; 15:491-498.

8. Reiber JHC, Serruys PW. Quantitative angiography. In: Marcus ML, Schelbert HR, Skorton DJ, eds. Cardiac Imaging, a Companion to Braunwalds Heart Disease. New York: Saunders, 1990:211-280.

9. Reiber JHC. Morphologic and densitometric quantitation of coronary stenoses; an overview of existing quantitation techniques. In: Reiber JHC, Serruys PW, eds. New Developments in Quantitative Coronary Angiography. Dordrecht: Kluwer Academic Publishers, 1990:34.

10. Beatt KJ, Luijten HE, de Feyter PJ, et al. Change in diameter of coronary artery segments adjacent to steno- sis after percutaneous transluminal coronary angioplasty: Failure of percent diameter stenosis measurement to reflect morphologic changes induced by balloon dilatation. J Am Coll Cardiol 1988; 12:315-323.

11. Gould KL, Lipscomb K, Hamilton GW. Physiologic basis for assessing critical stenoses: Instantaneous flow response and regional distribution during coronary hyperemia as measures of coronary flow reserve. Am J Cardiol $1974 ; 33: 87-94$

12. Nobuyoshi M, Kimura T, Nosaka $H$, et al. Restenosis after successful percutaneous transluminal coronary angioplasty: Serial angiographic follow-up of 299 patients. J Am Coll Cardiol 1988; 12:616-623.

13. Serruys PW, Luijten HE, Beatt KJ, et al. Incidence of restenosis after successful coronary angioplasty: A timerelated phenomenon. Circulation 1988; 77:361-371

14. Meyer J, Schmitz HJ, Kiesslich T, et al. Percutaneous transluminal coronary angioplasty in patients with stable and unstable angina pectoris: Analysis of early and late results. Am Heart J 1983; 106:973-980.

15. Fleck E, Dacian S, Dirschinger J, et al. Quantitative changes in stenotic coronary artery lesions during follow up after PTCA. (abstract) Circulation 1984; 70(Suppl II):II-176. 